\title{
Analysis on the Effect of Protective Equipment Training on Hospital Infection Managers
}

\author{
YongliZou ${ }^{1}$, Xiaohong Yang ${ }^{1}$, Ya Xu ${ }^{1}$, TingtingZhong ${ }^{2}$, Chi Liu ${ }^{1}$, Jiemin Liu ${ }^{1, *}$, Jixia Cao ${ }^{3}$ * \\ ${ }^{I}$ Guizhou Provincial People's Hospital, Guiyang 550002, China \\ ${ }^{2}$ Guizhou University of Traditional Chinese Medicine, Guiyang 550002, China \\ ${ }^{3}$ Army Military Transportation University, Transportation Unit Management Teaching and Research \\ Office, Tianjin 300161, China \\ *Corresponding Author.
}

\begin{abstract}
Objectives: To analyze the effect of personal protective equipment training on new hospital infection managers. Methods: Personnel are divided into two batches by region. Adopt a diversified training model to train all personnel, finally conduct practical assessments and issue certificates. Collect information through information technology, analyze questionnaires, and understand trainees' circumstances before and after the training. Each training batch has uniform teachers and the same training methods. Results: After the training, the trainees' proficiency in putting on and taking off protective equipment increased by $22.85 \%$, and ability to choose protective equipment according to different working environments increased by 22.04\%; $78.23 \%$ trainees believed that practical exercises should be emphasized. Taking off protective clothing was considered as the most difficult link in practical training $(91.13 \%)$, followed by putting on protective clothing $(70.43 \%) .96 .24 \%$ trainees believed that this training is helpful for future work. Conclusions: It is quite necessary to implement personal protective equipment training among new hospital infection managers; where, practical training, assessment, information-based questionnaire survey, expert theory teaching have achieved good results; the training helps reduce occupational exposure-induced hospital infection, and at the same time, avoids improper use of protective materials and waste.
\end{abstract}

Keywords: COVID-19, hospital infection manager, personal protection, training

\section{Background}

Coronavirus disease-19 (COVID-19) is a new acute respiratory infectious disease, which has become a major global public health event. Its infection source is mainly COVID-19 patients and asymptomatic patients. With infection during the incubation period, it is the most infectious within five days after the disease onset ${ }^{[1]}$. The main transmission way is through respiratory droplets and close contact. Contact with virus-contaminated items can also cause transmission. There is a possibility of aerosol transmission when one is exposed to high concentrations of aerosols for a long time in a relatively closed environment ${ }^{[1]}$. Personal protective equipment (PPE) offers an important protective barrier for medical staff, which can reduce the high infection rate caused by exposure, and plays an important role in isolation protection and reduced incidence of hospital infections. Letting medical staff proficiently master the standardized procedure for putting on and taking off PPE is the most effective measure to prevent infection ${ }^{[2,3]}$. According to the COVID-19 Epidemic Prevention and Control Plan (7th Edition) ${ }^{[4]}$, we clearly put forward the personal protection guidelines for specific populations. In order to do a good job of epidemic prevention and control and further strengthen the reserve of nosocomial infection managers, our hospital trained new nosocomial infection managers ${ }^{[5]}$. Putting on and taking off protective equipment is one training course in this training. We formulate the procedures and assessment standards for putting on and taking off protective equipment according to national guidelines ${ }^{[4]}$, and adopt diversified training methods to improve the ability to put on and take off protective equipment among hospital infection managers in our province, reduce hospital infections caused by occupational exposure. Good results are achieved, with the report made as follows: 


\section{Objectsand Methods}

\subsection{Research object}

373 new hospital infection managers reported by medical institutions across the province during March 1-11, 2021, who were trained in two batches.

\subsection{Evaluation method}

2.2.1 Training methods

Personnel are divided into two batches by region. A three-day closed training was given for each batch, and training for each batch was separated in two weeks. Diversified methods combining video demonstration, on-site theoretical lectures, evening intensive training, and practical training were adopted to train participants, and practical evaluations were finally made. After the trainees pass the theoretical and practical assessments, the Guizhou Provincial Hospital Infection Training Base will issue the "Guizhou Provincial Hospital Infection Management Post Training Certificate". We collected personal information and issued questionnaires through information technology like corporate WeChat, Sojump; provincial experts gave theoretical lessons; teaching videos on how to put on and take off protective equipment were shot in accordance with national regulations, and the videos and assessment standards were posted in the trainee WeChat group to facilitate their personal exercises; after the trainees learn to put on and take off the protective equipment, full-time training teacher would conduct one-to-one practical training ${ }^{[6]}$ and gave detailed guidance in the evening; finally, the trainees would be assessed, and those who do not meet the standards will be immediately arranged for retraining in a special area until the assessment is passed. At the graduation ceremony, the hospital leaders will conduct random tests on all the trainees who have passed the assessment. All trainees passing the assessment will be issued with certificates. Trainees are encouraged to return to respective medical institutions to further train the personnel of the unit with the acquired knowledge. Each batch adopts uniform teachers and the same training method.

\subsubsection{Process of putting on and taking off personal protective equipment}

(1) Process of putting on personal protective equipment: hand hygiene-wear medical protective mask-wear a disposable hat-wear inner gloves-wear protective clothing-wear outer gloves-wear goggles /protective visor-wear inner shoe covers (arranged into protective pants), boot covers-hand hygiene. (2)Sequence of taking off personal protective equipment: hand hygiene-enter No.1 Buffer Room-hand hygiene-take off goggles or protective visor-take off protective clothing, outer boots, outer gloves-hand hygiene-enter No.2 Buffer Room room- take off the inner shoe covers-take off the inner gloves-hand hygiene-remove the hat-take off the medical protective mask-hand hygiene (preferably wash hands with running water)-wear a medical surgical mask.

\subsubsection{Evaluation method}

Each batch of trainees will be assessed on the spot by uniform teacher against the assessment score sheet. A score of $\geq 80$ is considered as qualified, and a score of $<80$ is considered as unqualified. For those who fail the assessment, the assessment teacher will comment on the spot, and ask the trainees to further strengthen the practice for make-up exam until the assessment is passed and a certificate of qualification is issued. In the closing ceremony, 10 trainees will be randomly selected for on-site demonstrations, one-by-one comments and awards.

\subsubsection{Evaluation criteria}

(1) Whether the wearing sequence is correct; (2) Whether the skin is exposed; (3) Whether the removal sequence is correct; (4) Whether the removal process is contaminated.

\subsubsection{Points for scoring}

There are 15 steps to put on personal protective equipment, with 45 points in total. Each step corresponds to the corresponding score. Wearing medical protective masks according to the specification, proficiency in wearing protective clothing, checking protection tightness, and hand hygiene are the four key links, which take up a total of 
37 points. There are 10 steps to take off protective equipment, which are implemented in two areas and make up a total of 55 points. Where, taking off protective clothing in No.1 Buffer Room and taking off protective masks in No.2 Buffer Room are the key links, which take up a total of 31 points.

\subsection{Guarantee of epidemic prevention and control during the training period}

Fully enclosed management is adopted. When the trainees check in, they need receive an epidemiological investigation, provide a certificate of negative nucleic acid test within 7 days, and sign an epidemic prevention and control commitment. On the day of the training, an infrared ray was set up at the entrance to monitor the trainees' body temperature. Only those with normal body temperature can participate in the training, and medical surgical masks are required. The trainees' seats have a spacing of more than 1 meter. In accordance with the COVID-19 prevention and control requirements, the training venue should maintain clean environment, ventilated air, and fully open doors and windows during the meeting ${ }^{[7]}$ to ensure environmental safety. During the meeting, our hospital arranged emergency response personnel to treat and investigate the trainees with physical discomfort.

\subsection{Statistical methods}

The statistical software SPSS20.0 was used for statistical analysis of the data, and paired-samples ttest was used. The results show significant differences before and after training $(P<0.05)$, which is statistically significant.

\section{Results}

\subsection{Qualified status}

373 people participated in the training, of which 3 people did not participate in the practical operation assessment ( 2 in the first batch, 1 in the second batch), and the total number of assessed people was 370 . The average score of the first batch is 88.64 points; the average score of the second batch is 90.41 points; the total average score of the trainees in the two batches is 89.5 points, and 370 assessment participants are all qualified.

\subsection{Basic information}

According to the requirements of this training, by consulting relevant documents and guidelines, we design questionnaires, distribute questionnaires through the information platform, and fill in the questionnaires. Except for one person who did not fill in the first batch, the rest completed the questionnaires, and a total of 372 people participated in questionnaire survey before and after training.

The trainees registered through the corporate WeChat so that we collected trainees' information and learned about the trainees' knowledge and mastery of protective equipment at the same time. The questionnaire conducts survey mainly from six aspects, see: Table 1 .

After the training, the questionnaire was distributed through the WeChat group of the trainees to understand trainees' feedback after the training, and the survey was conducted mainly from eight aspects, as shown in Table 2. 
Table 1 Statistics of pre-training surveys

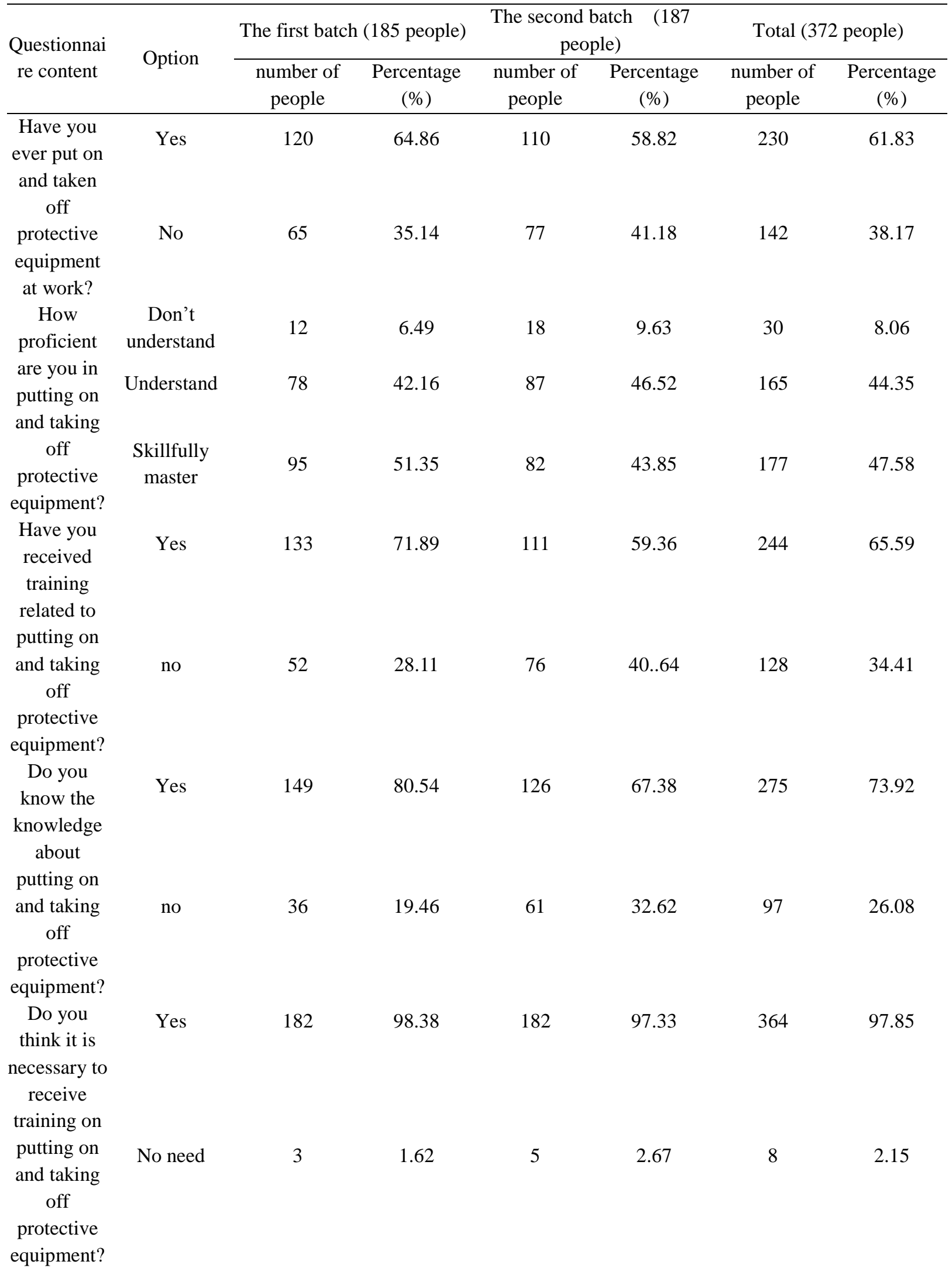




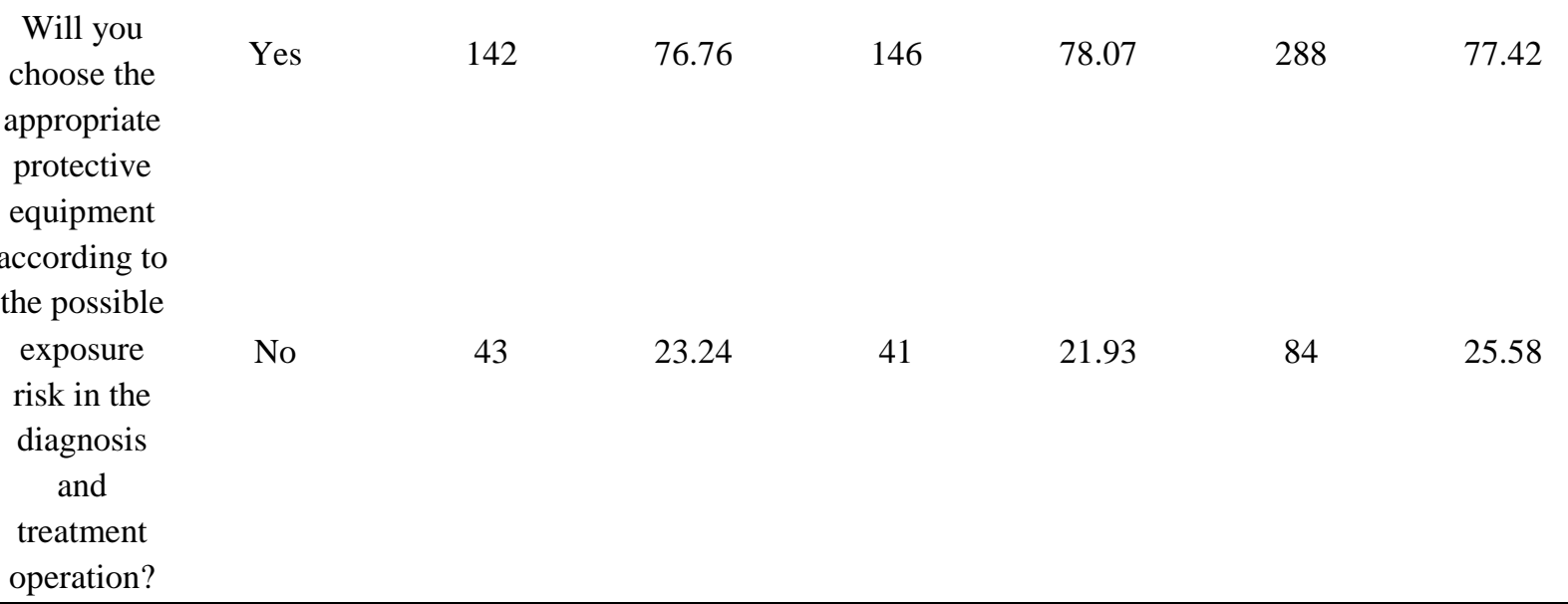

Table 2 Statistics of surveys after personnel training

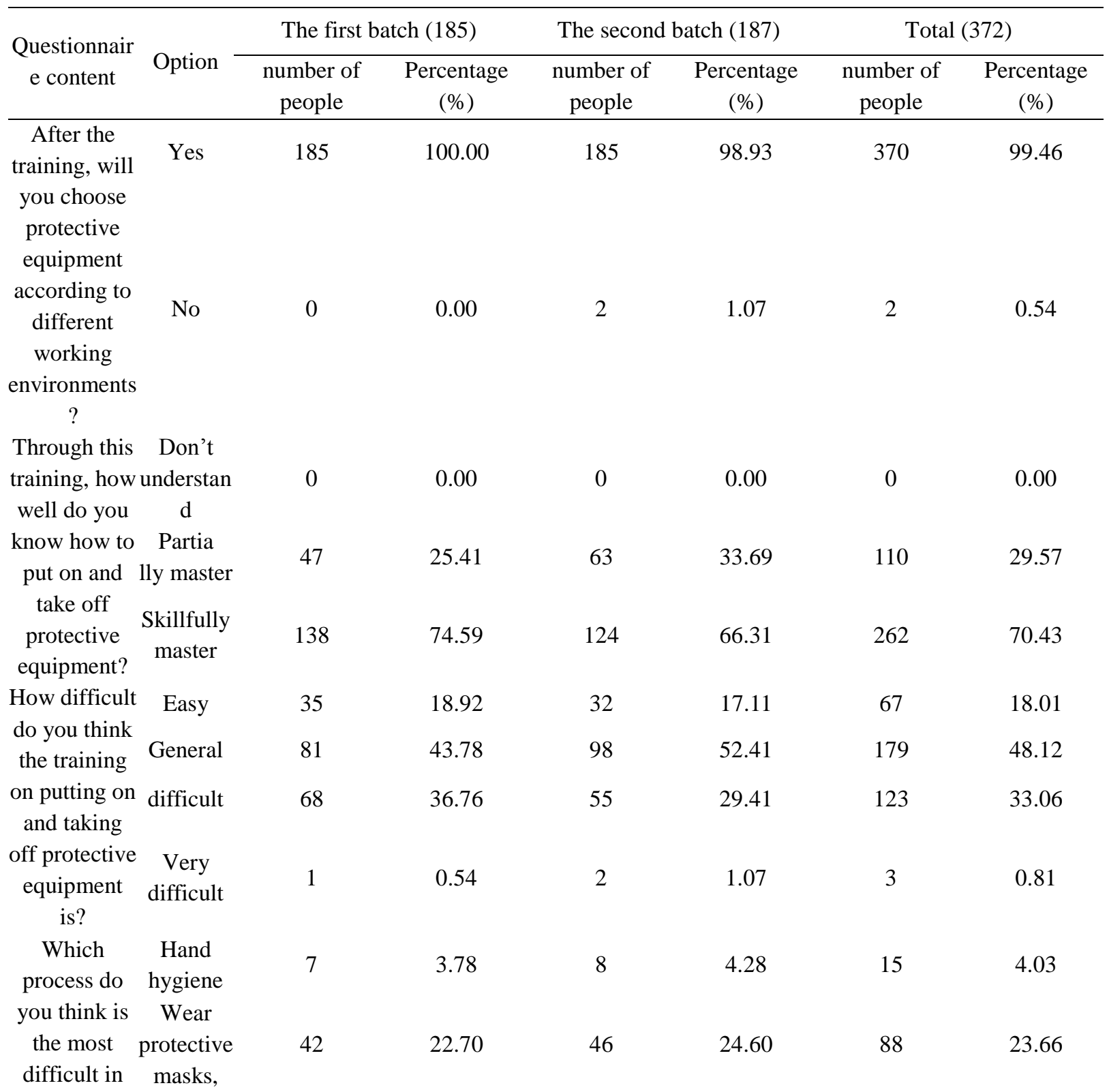


putting on

protective inner and

equipment? outer

gloves

Wear

protective

135

clothing

Wear

goggles

Hand

hygiene

Take off

$\begin{array}{ll}\text { Which } & \text { goggles } \\ \text { Take off }\end{array}$

process do

you think is

the most

difficult in

taking off

protective

equipment? rotective

clothing

Take off

protective

mask

Take off

shoe

covers,

gloves,

hats

Do you think the key

Yes

content and

time

allocation of

this training

on putting on

and taking

off protective

equipment is

reasonable?

No

(If

unreasonable

, please

explain the

reasons and

give

suggestions)

What links Theory

do you think teaching

87

47.03

72

38.50

159

42.74

should be Practical

highlighted training

141

76.22

131

70.05

272

73.12

in this Practical

training on exercise

142

76.76

149

79.68

291

78.23

putting on

and taking

test

61

32.97

43

22.99

104

27.96 
off protective

equipment?

(Multiple

choice

questions)

Do you think Little

this training help

on putting on General

0

6

0.00

3

3.24

5

1.60

3

and taking

off protective

equipment

will help you

in your

future work?
Very

useful

179

96.76

179

95.72

358

96.24

\subsection{Questionnaire results}

Seen from training results of the two batches, the trainees' proficiency in putting on and taking off protective equipment was $47.58 \%$ before the training and $70.43 \%$ after the training. A paired-samples $t$ test was performed on the proficiency before and after the training, and the results showed a significant difference in the proficiency before and after the two trainings (the first batch: $\mathrm{t}=5.638 \mathrm{P}<0.05$, the second batch: $\mathrm{t}=5.903 \mathrm{P}<0.05$ ), indicating obvious training effect.

Ability to choose protective equipment according to different working environments increased from $77.42 \%$ before training to $99.46 \%$ after training, an increase of $22.04 \%$, indicating that the trainees have improved ability in choosing protective equipment reasonably at work after training.

96.24\% trainees believe that this training on putting on and taking off protective equipment is helpful to their future work.

In the process of wearing protective equipment, $70.43 \%$ trainees thought that putting on protective clothing was the most difficult, and $23.66 \%$ trainees thought that putting on protective masks, hats, and inner and outer gloves was the most difficult.

In the process of taking off protective equipment, $91.13 \%$ trainees thought that taking off protective clothing was the most difficult, and $2.96 \%$ trainees thought that taking off shoe covers, gloves, and hats was the most difficult.

Regarding links that should be highlighted in the training of putting on and taking off protective equipment: $78.23 \%$ trainees choose practical exercise, and $73.12 \%$ trainees choose practical training.

\section{Discussion}

At present, the generally recognized transmission way of COVID-19 is droplet transmission and close contact transmission. There is no direct evidence for confirming other transmission modes and their conditions. Considering the occurrence of related cases, the possibility of other transmission routes cannot be ruled out ${ }^{[8]}$. Putting on and taking off personal protective equipment is an effective preventive measure to cut off the infection transmission route and prevent the transmission of infection from patients and carriers to others ${ }^{[9]}$. During the COVID-19 epidemic, the role of protective clothing and protective masks as "armor" should be brought into full play. 
The Technical Guidelines for the Prevention and Control of COVID-19 Infection in Medical Institutions (Second Edition) specifically proposes that primary medical and health institutions, social medical institutions, specialist hospitals, and Chinese medicine hospitals, etc., should pay more attention to infection prevention and control work, sort out the existing weak links in the institutions, and continuously carry out full-staff training ${ }^{[10]}$. Most trainees come from the abovehospital. This training should keep pace with the times, and actively respond to the country's prevention and control needs. New hospital infection managers should be trained to skillfully master the knowledge, methods and skills for the prevention and control of COVID-19 infections, thus providing an important guarantee for the epidemic prevention and control in our province.

The protective equipment training should adopt diversified methods such as: video demonstration, theory teaching, closed management, one-to-one intensive training in the evening, one-to-one assessment on the spot, issuance of qualified certificates, random tests and operation evaluation, etc. which achieved good results in this training. The trainees skillfully mastered how to put on and take off protective equipment and had significantly improved ability to correctly select personal protective equipment. Only by proficiently mastering and standardizing the use of protective equipment can we achieve the prevention and control goal of "zero infection" among medical staff at all levels, reduce the occurrence of nosocomial infections, avoid excessive personal protection and waste of protective materials.

Putting on and taking off protective equipment is a practical process. Trainees hope that through practical training and exercises, they can strengthen communication with the trainer to improve their prevention and control capabilities, protect patients and themselves, thus better curing patients.

Information-based questionnaire surveys facilitate the collection and result analysis of questionnaires, so that we can find out medical staff's difficulties in putting on and taking off protective equipment, strengthen training with focus to achieve a good training effect, which is also beneficial to the future rationalization and improvement of the protection process.

In summary, during the COVID-19 epidemic, personal protection serves as the "armor" in medical staff protection, which must be mastered by everyone. Through the use of diversified training methods, this research found that practical training and intensive exercises play a major role in achieving good results. Through one-to-one guidance, medical staff can master the skills of putting on and taking off personal protective equipment as quickly as possible, thus reducing the risk of medical staff infection. This research also pays attention to the medical staff feedback information after training, which will facilitate the continuous improvement of our training in the next step.

\section{References}

[1] "General office of the national health commission," Notice on Issuing the COVID-19 Diagnosis and Treatment Plan (Trial Eighth Edition, Revised Edition),vol. 4, pp. 14, 2021. http://www.nhc.gov.cn/yzygj/s7653p/202104/7de0b3837c8b4606a0594aeb0105232b.shtml

[2] "The medical care and hospital administration of the national health commission of the people's republic of China," Notice of the General Office of the National Health Commission on Strengthening the Management of Medical Protective Products During the Epidemic, vol. 2, pp. 4, 2020.

[3] P. Chen, H.Y. Zhang, W. Chen, et al.,"Monitoring and evaluation of medical personnel's operation errors in taking off protective equipment," Chinese Journal of Infection Control, vol. 19, no. 11, pp. 1033-1036, 2020. DOI: 10.12138 /j. issn. 1671-9638.

[4] "Bureau of disease control and prevention," COVID-19 Prevention and Control Plan (Seventh Edition), vol. 9, pp. 15, 2020.

[5] "Guizhouprovincial health commission," Notice of the Provincial Health Commission on Continuing the On-the-job Training of Hospital Infection Management Professionals, vol. 2, pp. 25, 2021.

[6] L.P. Shi, J. Song, “Analysis and countermeasures to the problem of putting on and taking off protective 
equipment among medical staff in response to COVID-19," World Latest Medicine Information, vol. 20, no. 74, pp. 250-252, 2020.

[7] "The State Council's joint prevention and control mechanism for the COVID-19 epidemic," Notice on the Scientific and Accurate Prevention and Control of COVID-19 Epidemic in Accordance with the Law, vol. 2, pp. 5, 2020.

[8] J.M. Yuan, H. Ren, Y. Sun, et al.,"Analysis and thinking on the transmission route of COVID-19 in 2019,” Journal of Xi'an Jiaotong University, vol. 3, no. 3, pp. 1-3, 2020.

[9] Y.X. Qiu, B.L. Ye, Q.F. Dong, et al.,"Research on effective training methods for personal protection against COVID-19," Chinese Journal of Infection Control, vol. 19, no. 6, pp. 513-517, 2020. DOI: 10.12138/j. issn. 1671-9638. 20206812.

[10] Notice of the General Office of the National Health Commission on Issuing the Technical Guidelines for the Prevention and Control of COVID-19 Infection in Medical Institutions (Second Edition), vol. 4, pp. 14, 2021. 